\title{
On the degeneracy of atomic states within exact-exchange (spin-) density functional theory
}

\author{
S. Pittalis, S. Kurth and E.K.U. Gross \\ Institut für Theoretische Physik, Freie Universität Berlin, Arnimallee 14, D-14195 Berlin, Germany
}

\begin{abstract}
The problem of degenerate ground states of open-shell atoms is investigated in spin-restricted and unrestricted density functional theory using the exact exchange energy functional. For the spinunrestricted case, spurious energy splittings of the order of 2 to $3 \mathrm{kcal} / \mathrm{mol}$ are found for atoms of the second and third period which is larger than the splittings obtained from recently proposed approximate exchange functionals depending explicitly on the current density. In remarkable contrast, for spin-restricted calculations the degeneracy of different atomic ground states is recovered to within less than $0.6 \mathrm{kcal} / \mathrm{mol}$.
\end{abstract}

\section{INTRODUCTION}

The Hohenberg-Kohn [1] and Kohn-Sham [2] theorems of density functional theory (DFT), which were originally established for non-degenerate ground states, may be extended to degenerate ground states as well [3, 4]. These degenerate ground states lead to a set of different ground state densities and the exact energy functional yields the same ground state energy for all these densities. It has long been known, however, that common approximations do not yield the same total energies [5, 6, 7, 8]. In a systematic investigation of this problem Baerends and coworkers [9] showed that for states with different total magnetic quantum number, $M_{L}$, spurious energy splittings of up to $5 \mathrm{kcal} / \mathrm{mol} \mathrm{re-}$ sult from generalized gradient approximations (GGA's). Even larger ones are observed for the meta-GGA's [10].

The problem has attracted renewed interest recently. Becke has proposed an approach for constructing exchange-correlation functionals with an increased ability to reproduce the degeneracy of atomic states [11]. The essential idea is to enforce the proper description of the Fermi (or exchange) hole curvature [12] in the approximation of the exchange-correlation energy functional [13]. As a consequence, the paramagnetic current density appears explicitly in the expression of the corresponding functional [14]. This improves the description of the atomic degeneracy [11] of states carrying different paramagnetic current densities.

Along the line of Becke's approach, Maximoff et al [15] have modified the GGA of Perdew, Burke, and Ernzerhof (PBE) to a form explicitly dependening on the current density. In this way they successfully reduced the previous spurious energy splittings. Actually, they have weakened Becke's suggestion by improving not the exchange hole curvature at all points in space, but rather its average.

More recently, Tao and Perdew [10] have employed ideas of the current-DFT framework of Vignale and Rasolt [16]. They constructed a current-dependent correction to GGA and meta-GGA functionals and their results again suggest that some improvements for the energy splittings can be achieved.

In this work we test the performance of the exact exchange energy functional using the Optimized Effective
Potential method [17, 18] for the problem of degenerate ground states. In particular, we analyze an interesting aspect of the degeneracy problem related to the additional degrees of freedom introduced by going from the original DFT formulation of Hohenberg, Kohn and Sham to the spin-DFT (SDFT) formalism of von Barth and Hedin [19]. As a consequence of this additional variational freedom, lower total energies are obtained in SDFT than in corresponding spin-restricted (DFT) calculations. At the same time, however, the spurious energy splittings are increased for the states of different $M_{L}$. In this work we only consider densities represented by single Slater determinants of Kohn-Sham orbitals. The general formalism to deal with densities which can only be described as weighted sum of several determinantal densities is discussed in Refs. [20, 21].

In the following we recall the basic ideas of the Optimized Effective Potential method and briefly compare the resulting equations in the DFT and SDFT framework. We then give some details on our numerical implementation along with the resulting energy splittings for the exact-exchange functional. Our findings are compared with results from other approximations discussed in the literature before we draw our conclusions.

\section{SPIN RESTRICTED AND UNRESTRICTED KOHN-SHAM SCHEMES}

In this Section we briefly review the basic equations of spin-restricted and spin-unrestricted density functional theory. We then focus on orbital-dependent approximations to the exchange-correlation energy functional and discuss, for a given orbital functional, the relation between the corresponding exchange-correlation potentials in the restricted (DFT) and the unrestricted (SDFT) formalisms.

In the unrestricted SDFT formalism, the total energy $E$ of a system of interacting electrons is a functional of the two spin densities $\rho_{\sigma}(\mathbf{r})(\sigma=\uparrow, \downarrow)$ :

$$
\begin{aligned}
& E\left[\rho_{\uparrow}, \rho_{\downarrow}\right]=T_{s}\left[\rho_{\uparrow}, \rho_{\downarrow}\right] \\
& \quad+\int \mathrm{d}^{3} r v_{0}(\mathbf{r}) \rho(\mathbf{r})+U[\rho]+E_{x c}\left[\rho_{\uparrow}, \rho_{\downarrow}\right]
\end{aligned}
$$


where

$$
T_{s}\left[\rho_{\uparrow}, \rho_{\downarrow}\right]=\sum_{\sigma=\uparrow, \downarrow} \sum_{j}^{N_{\sigma}} \int \mathrm{d}^{3} r \varphi_{j \sigma}^{*}(\mathbf{r})\left(-\frac{\nabla^{2}}{2}\right) \varphi_{j \sigma}(\mathbf{r})
$$

is the non-interacting kinetic energy and $N_{\sigma}$ is the number of electrons with spin $\sigma . v_{0}(\mathbf{r})$ is an external, electrostatic potential and

$$
\rho(\mathbf{r})=\rho_{\uparrow}(\mathbf{r})+\rho_{\downarrow}(\mathbf{r})
$$

is the total electronic density. The classical electrostatic (Hartree) interaction energy is given by

$$
U[\rho]=\frac{1}{2} \int \mathrm{d}^{3} r \int \mathrm{d}^{3} r^{\prime} \frac{\rho(\mathbf{r}) \rho\left(\mathbf{r}^{\prime}\right)}{\left|\mathbf{r}-\mathbf{r}^{\prime}\right|}
$$

and $E_{x c}$ is the exchange-correlation energy functional which has to be approximated in practice. The singleparticle orbitals $\varphi_{j \sigma}(\mathbf{r})$ in Eq. (2) are solutions of the Kohn-Sham equation [19]

$$
\left(-\frac{\nabla^{2}}{2}+v_{s \sigma}(\mathbf{r})\right) \varphi_{j \sigma}(\mathbf{r})=\varepsilon_{j \sigma} \varphi_{j \sigma}(\mathbf{r})
$$

where $j$ is a collective index for the one-electron quantum numbers except spin. The effective single particle potential for spin $\sigma$ is given by

$$
v_{s \sigma}(\mathbf{r})=v_{0}(\mathbf{r})+v_{H}(\mathbf{r})+v_{x c \sigma}(\mathbf{r})
$$

with the Hartree potential

$$
v_{H}(\mathbf{r})=\int \mathrm{d}^{3} r^{\prime} \frac{\rho\left(\mathbf{r}^{\prime}\right)}{\left|\mathbf{r}-\mathbf{r}^{\prime}\right|}
$$

and the exchange-correlation potential

$$
v_{x c \sigma}(\mathbf{r})=\frac{\delta E_{x c}\left[\rho_{\uparrow}, \rho_{\downarrow}\right]}{\delta \rho_{\sigma}(\mathbf{r})} .
$$

The self-consistency cycle is closed by computing the spin densities via

$$
\rho_{\sigma}(\mathbf{r})=\sum_{j=1}^{N_{\sigma}}\left|\varphi_{j \sigma}(\mathbf{r})\right|^{2}
$$

where the sum runs over the occupied orbitals.

The unrestricted Kohn-Sham scheme of SDFT immediately reduces to the restricted scheme of DFT if one considers exchange-correlation functionals which only depend on the total electronic density of Eq. (3) as envisioned in the original Hohenberg-Kohn theorem [1]. Then the exchange-correlation potential is

$$
v_{x c}(\mathbf{r})=\frac{\delta E_{x c}[\rho]}{\delta \rho(\mathbf{r})}
$$

and both $v_{x c}$ and the total effective potential $v_{s}$ are independent of the spin index $\sigma$. Note that in spinte of the spin-independence of $v_{s}$ and $v_{x c}$, the Kohn-Sham orbitals, being proper fermionic single-particle orbitals, still carry a spin-dependence.

Of course, the exchange-correlation energy functional needs to be approximated in practice. Popular approximations like the local density approximation (LDA) or generalized gradient approximations (GGA's) use an approximate form of $E_{x c}$ which explicitly depends on the density (in DFT) or on the spin-densities (in SDFT), i.e., different forms of the functional are required in DFT and SDFT. However, if one considers functionals which explicitly depend on the single-particle orbitals rather than the (spin-)density, one and the same orbital functional may be used either in the DFT or in the SDFT framework. The difference is the implicit dependence of the Kohn-Sham orbitals on the corresponding basic variables: in DFT they are implicit functionals of the total particle density only, while in SDFT the orbitals are implicit functionals of the spin densities.

For orbital functionals, the calculation of the exchange-correlation potential is somewhat more complicated than for explicit density functionals and is achieved with the so-called Optimized Effective Potential Method (OEP) [17, 18]. For a review of the method the reader is referred to Ref. [22]. The OEP method leads to an integral equation for the exchange-correlation potential. For simplicity, we consider approximations of $E_{x c}$ that are functionals of the occupied orbitals only. The OEP integral equation can then be written in compact notation (in SDFT)

$$
\sum_{j=1}^{N_{\sigma}}\left(\psi_{j \sigma}^{*}(\mathbf{r}) \varphi_{j \sigma}(\mathbf{r})+\text { c.c. }\right)=0 .
$$

Here we have defined the orbital shifts

$$
\psi_{j \sigma}^{*}(\mathbf{r})=\int \mathrm{d}^{3} r^{\prime} \varphi_{j \sigma}^{*}\left(\mathbf{r}^{\prime}\right)\left(v_{x c \sigma}\left(\mathbf{r}^{\prime}\right)-u_{x c j \sigma}\left(\mathbf{r}^{\prime}\right)\right) G_{S j \sigma}\left(\mathbf{r}^{\prime}, \mathbf{r}\right)
$$

where $G_{S j \sigma}$ is the Green function of the Kohn-Sham system

$$
G_{S j \sigma}\left(\mathbf{r}^{\prime}, \mathbf{r}\right)=\sum_{\substack{k=1 \\ \varepsilon_{k \sigma} \neq \varepsilon_{j \sigma}}}^{\infty} \frac{\varphi_{k \sigma}^{*}\left(\mathbf{r}^{\prime}\right) \varphi_{j \sigma}(\mathbf{r})}{\varepsilon_{j \sigma}-\varepsilon_{k \sigma}}
$$

and

$$
u_{x c j \sigma}(\mathbf{r})=\frac{1}{\varphi_{j \sigma}^{*}(\mathbf{r})} \frac{\delta E_{x c}}{\delta \varphi_{j \sigma}(\mathbf{r})} .
$$

In a series of steps [22, 23], the OEP equation can be transformed to 


$$
v_{x c \sigma}(\mathbf{r})=\frac{1}{2 \rho_{\sigma}(\mathbf{r})} \sum_{j=1}^{N_{\sigma}}\left[\left|\varphi_{j \sigma}(\mathbf{r})\right|^{2}\left(u_{x c j \sigma}(\mathbf{r})+\left(\bar{v}_{x c j \sigma}-\bar{u}_{x c j \sigma}\right)\right)-\nabla \cdot\left(\psi_{j \sigma}^{*}(\mathbf{r}) \nabla \varphi_{j \sigma}(\mathbf{r})\right)\right]+c . c .
$$

where

$$
\bar{v}_{x c j \sigma}=\int \mathrm{d}^{3} r \varphi_{j \sigma}^{*}(\mathbf{r}) v_{x c \sigma}(\mathbf{r}) \varphi_{j \sigma}(\mathbf{r})
$$

and

$$
\bar{u}_{x c j \sigma}=\int \mathrm{d}^{3} r \varphi_{j \sigma}^{*}(\mathbf{r}) u_{x c j \sigma}(\mathbf{r}) \varphi_{j \sigma}(\mathbf{r}) .
$$

Similar expressions can, of course, be obtained for the spin-restricted case. The OEP equation analogous to Eq. (11) reads

$$
\sum_{\sigma=\uparrow, \downarrow} \sum_{j=1}^{N_{\sigma}}\left(\tilde{\psi}_{j \sigma}^{*}(\mathbf{r}) \varphi_{j \sigma}(\mathbf{r})+\text { c.c. }\right)=0 .
$$

where the modified orbital shifts $\tilde{\psi}_{j \sigma}$ are defined in analogy to Eq. (12) with $v_{x c \sigma}$ being replaced by $v_{x c}$. Applying the same steps as in the SDFT case, the OEP equation of DFT transforms to

$$
\begin{aligned}
& v_{x c}(\mathbf{r})=\frac{1}{2 \rho(\mathbf{r})} \\
& \sum_{\sigma=\uparrow, \downarrow} \sum_{j=1}^{N_{\sigma}}\left[\left|\varphi_{j \sigma}(\mathbf{r})\right|^{2}\left(u_{x c j \sigma}(\mathbf{r})+\left(\tilde{v}_{x c j \sigma}-\bar{u}_{x c j \sigma}\right)\right)\right. \\
& \left.\quad-\nabla \cdot\left(\tilde{\psi}_{j \sigma}^{*}(\mathbf{r}) \nabla \varphi_{j \sigma}(\mathbf{r})\right)\right]+c . c .
\end{aligned}
$$

where $\tilde{v}_{x c j \sigma}$ is defined as $\bar{v}_{x c j \sigma}$ in Eq. (16) except that $v_{x c \sigma}$ is again replaced by $v_{x c}$. The DFT exchangecorrelation potential (19) can be written as a weighted average of potentials for the different spin channels

$$
v_{x c}(\mathbf{r})=\frac{\rho_{\uparrow}(\mathbf{r}) \tilde{v}_{x c \uparrow}(\mathbf{r})+\rho_{\downarrow}(\mathbf{r}) \tilde{v}_{x c \downarrow}(\mathbf{r})}{\rho_{\uparrow}(\mathbf{r})+\rho_{\downarrow}(\mathbf{r})}
$$

where

$$
\begin{aligned}
& \tilde{v}_{x c \sigma}(\mathbf{r})=\frac{1}{2 \rho_{\sigma}(\mathbf{r})} \\
& \sum_{j=1}^{N_{\sigma}}\left[\left|\varphi_{j \sigma}(\mathbf{r})\right|^{2}\left(u_{x c j \sigma}(\mathbf{r})+\left(\tilde{v}_{x c j \sigma}-\bar{u}_{x c j \sigma}\right)\right)\right. \\
& \left.\quad-\nabla \cdot\left(\tilde{\psi}_{j \sigma}^{*}(\mathbf{r}) \nabla \varphi_{j \sigma}(\mathbf{r})\right)\right]+c . c .
\end{aligned}
$$

Eq. (20) shows how, in the spin-restricted case, the spin-up and spin-down channels mix to form the spinindependent exchange-correlation potential.
Eqs. (11) or 19] can be solved iteratively along with the corresponding Kohn-Sham equations in a selfconsistent fashion. Due to the presence of the unoccupied Kohn-Sham orbitals in the definition of the orbital shifts (see Eqs. (12) and (13)), the full numerical solution of the OEP integral equation is nontrivial. In the original paper [18], solutions were presented for atomic systems with spherical symmetry. Much later, it has also been solved for systems with lower symmetry such as molecules [24, 25] and solids [26]. Recently, an iterative algorithm for the solution of the OEP integral equation based on the orbital shifts has been implemented [27, 28].

In what follows we do not attempt a solution of the full OEP equation but rather use an approximation suggested by Krieger, Li and Iafrate [29] which has been found to be rather accurate in many situations. In this so-called KLI approximation, the terms containing the orbital shifts on the r.h.s. of Eqs. (11) or (19) are neglected completely. The KLI approximation may be substituted by a slightly more elaborate one known as Common Energy Denominator Approximation (CEDA) [30] or Localized Hartree-Fock (LHF) approximation [31]. However, it has been found that CEDA and KLI total energies are extremely close for atoms [32]. Moreover, for the atoms studied in this work we expect that KLI and CEDA results are very similar also for the currentcarrying states since in most cases (from boron to magnesium) the current-carrying orbitals enter the expressions for the KLI and CEDA potentials in exactly the same way.

The OEP equations given above are valid for any form of the exchange-correlation functional $E_{x c}$ which depends on the occupied orbitals only. In this work we use the exact exchange functional

$$
\begin{aligned}
& E_{x}=-\frac{1}{2} \sum_{\sigma=\uparrow, \downarrow} \sum_{j, k=1}^{N_{\sigma}} \\
& \quad \int \mathrm{d}^{3} r \int \mathrm{d}^{3} r^{\prime} \frac{\varphi_{j \sigma}^{*}(\mathbf{r}) \varphi_{k \sigma}^{*}\left(\mathbf{r}^{\prime}\right) \varphi_{j \sigma}\left(\mathbf{r}^{\prime}\right) \varphi_{k \sigma}(\mathbf{r})}{\left|\mathbf{r}-\mathbf{r}^{\prime}\right|}
\end{aligned}
$$

which is nothing but the Fock term of Hartree-Fock theory evaluated with Kohn-Sham orbitals. In our calculations the correlation energy is neglected completely.

\section{NUMERICAL RESULTS}

In this Section we present our numerical results on the degeneracy problem of open-shell atomic ground states. 
It is well-known that standard approximations like LDA or GGA do not give the same, degenerate ground state energies for different open-shell configurations. This is due to the fact that the densities of these ground states are different, leading to different Kohn-Sham potentials derived from these densities and therefore also to different total energies [9]. Recently, this problem has attracted renewed interest [10, 11, 15] where approximate functionals depending on the current-density have been suggested which, while not solving the problem completely, at least reduce the energy splittings between different configurations significantly. Here we investigate the problem at the exact-exchange level, both in DFT and SDFT.

Although the (interacting) Hamiltonian of an atom has spherical symmetry, the ground state densities of open-shell atoms typically are not spherical. However, for any of the possible degenerate ground states one can always find an axis for which the corresponding density exhibits cylindrical symmetry and we choose this axis as the $z$-axis of our coordinate system. We seek a KohnSham single-particle potential with the same cylindrical symmetry. Then the magnetic quantum number $m$ is a good quantum number to characterize the Kohn-Sham orbitals. We can then perform self-consistent calculations by specifying how many orbitals with $m=0,1, \ldots$ be occupied for each spin channel and then keep this configuration fixed throughout the self-consistency cycle. For example, for the boron atom, one configuration has all spin-up electrons and the two spin-down electrons in $m=0$ states while in another configuration one of the spin-up electrons is required to occupy an $m=1$ state with the other occupations unchanged. In this way current-carrying and zero-current states can be considered.

We have developed an atomic code for DFT and SDFT calculations in a basis set representation, assuming cylindrical symmetry of the Kohn-Sham potential. As basis functions we use Slater-type basis functions for the radial part multiplied with spherical harmonics for the angular part. We use the quadruple zeta basis sets (QZ4P) of Ref. [33] for the Slater functions.

We have tested our code by computing the total energies of spherically symmetric atoms of the first and second row of the periodic table in exchange-only KLI approximation and compared with results from accurate, fully numerical codes available in the literature [22, 29, 34]. Our code reproduces these energies to within a maximum deviation of $0.3 \mathrm{Kcal} / \mathrm{mol}$ and an average deviation of $0.1 \mathrm{Kcal} / \mathrm{mol}$ for the first-row atoms and to within a maximum deviation of $0.9 \mathrm{Kcal} / \mathrm{mol}$ and an average deviation of $0.5 \mathrm{Kcal} / \mathrm{mol}$ for the second row. As a more relevant estimate of the accuracy of our calculations we have also computed the energy splittings between different configurations in LSD. Our results reproduce those reported in Ref. [11] with a deviation of less than $0.02 \mathrm{kcal} / \mathrm{mol}$.

We then calculated self-consistent total energies for

\begin{tabular}{|c|c|c|c|c|}
\hline 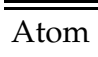 & $\overline{\Delta_{j B R}{ }^{a}}$ & $\overline{\overline{\Delta_{j P B E}}}{ }^{b}$ & 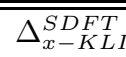 & 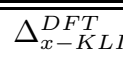 \\
\hline B & 0.62 & -0.4 & 1.66 & 0.06 \\
\hline C & 0.69 & -0.7 & 1.58 & 0.06 \\
\hline $\mathrm{O}$ & 1.23 & -0.6 & 2.36 & 0.55 \\
\hline $\mathrm{F}$ & 1.51 & -0.6 & 2.32 & 0.40 \\
\hline $\mathrm{Al}$ & 0.96 & 0.2 & 1.68 & 0.04 \\
\hline $\mathrm{Si}$ & 0.84 & -0.1 & 1.76 & 0.05 \\
\hline$S$ & 1.95 & 0.7 & 3.04 & 0.34 \\
\hline $\mathrm{Cl}$ & 1.73 & 0.3 & 3.15 & 0.25 \\
\hline
\end{tabular}

${ }^{a}$ current-dependent exchange functional of Ref. [11]

${ }^{b}$ current-dependent exchange functional of Eq.(17) of Ref. [15]

TABLE I: Spurios energy splittings, $\Delta=E\left(\left|M_{L}\right|=1\right)-$ $E\left(M_{L}=0\right)$ in $\mathrm{kcal} / \mathrm{mol}$. All the columns, but the last one, refer to spin-unrestricted calculations

the different configurations of open-shell atoms. Table 【 shows the energy differences (spurious energy splittings) between Kohn-Sham Slater determinants with total magnetic quantum number $\left|M_{L}\right|=1$ and $M_{L}=0$ in $\mathrm{kcal} / \mathrm{mol}$ of our exchange-only KLI calculations of DFT and SDFT. For comparison we also list the results of the current-dependent exchange-only functionals of Refs. [11] (denoted jBR) and [15] (denoted jPBE) in the first and second column, respectively. As can be seen, our SDFT results for the exact-exchange functional lead to larger splittings than both the jBR and the jPBE functionals. The idea behind the construction of these functionals is to improve the exchange-hole curvature by inclusion of the orbital paramagnetic current density. Since in our calculations we have used the exact exchange functional (and therefore also the correct exchange hole curvature) the success of the jBR and jPBE functionals in reducing the energy splittings might actually be due to an overcorrection of their parent functionals.

The most remarkable results of our calculations are the energy splittings obtained with a pure DFT (i.e., spin-restricted) calculation using the exact exchange functional (last column of Table【). These spurious splittings are in most cases more than an order of magnitude smaller than the corresponding SDFT results, therefore basically reproducing the exact degeneracy of the different ground-state configurations. Of course, due to the additional variational degree of freedom, total energies in SDFT are always lower than corresponding DFT results. The price to be paid for this improvement, however, are the unphysically increased energy splittings.

\section{CONCLUSIONS}

In this work we have calculated the spurious energy splittings between atomic states of different quantum 
number $M_{L}$ using the exact-exchange functional. We have employed the KLI approximation to compute the Kohn-Sham exchange potential and found that for spinunrestricted calculations the splittings are between 1 and $3 \mathrm{kcal} / \mathrm{mol}$ for the atoms of the second and third period. Somewhat surprisingly, these are larger than the splittings reported with approximate exchange functionals which include the paramagnetic current density as an input parameter [11, 15].

However, if the exact exchange functional is used in a spin-restricted DFT calculation, the spurious energy splittings are reduced significantly, the largest one being of the order of $0.5 \mathrm{kcal} / \mathrm{mol}$. One might speculate that the remaining splittings are due to the KLI approximation and could be further reduced if the full OEP equations for the exchange potential are solved.

\section{Acknowledgements}

We gratefully acknowledge financial support through the Deutsche Forschungsgemeinschaft Priority Program 1145 "First-Principles Methods", through the EU Network of Excellence NANOQUANTA and through the EU Research and Training Network EXCITING.
[1] P. Hohenberg and W. Kohn, Phys. Rev. 136, B864 (1964).

[2] W. Kohn and L.J. Sham, Phys. Rev. 140, A1133 (1965).

[3] W. Kohn, in Highlights of Condensed Matter Theory, ed. by F. Bassani, F. Fumi, M.P. Tosi (North-Holland, Amsterdam, 1985).

[4] R.M. Dreizler and E.K.U. Gross, Density Functional Theory (Springer, Berlin, 1990).

[5] T. Ziegler, A. Rauk, and E.J. Baerends, Theoret. Chim. Acta 43, 261 (1977).

[6] U. von Barth, Phys. Rev. A 20, 1693 (1979).

[7] F. Kutzler and G.S. Painter, Phys. Rev. B 43, 6865 (1991).

[8] R. Merkle, A. Savin, and H. Preuss, J. Chem. Phys. 97, 9216 (1992).

[9] E. J. Baerends, V. Branchadell, and M. Sodupe, J. Chem. Phys. 265, 481 (1997).

[10] J. Tao and J.P. Perdew, Phys. Rev. Lett. 95, 196403 (2005).

[11] A. Becke, J. Chem. Phys. 117, 6935 (2002).

[12] J.F. Dobson, J. Chem. Phys. 98, 8870 (1993).

[13] A. Becke, Can. J. Chem. 74, 995 (1996).

[14] A.D. Becke and M.R. Roussel, Phys. Rev. A 39, 3761 (1989).

[15] S. Maximoff, M. Ernzerhof, and G.E. Scuseria, J. Chem. Phys. 97, 9216 (1992).

[16] G. Vignale and M. Rasolt, Phys. Rev. B 37, 10685 (1988).

[17] R. Sharp and G. Horton, Phys. Rev. 90, 317 (1953).

[18] J.D. Talman and W.F. Shadwick, Phys. Rev. A 14, 36 (1976).

[19] U. von Barth and L. Hedin, J. Phys. C 5, 1629 (1972).

[20] C.A. Ullrich and W. Kohn, Phys. Rev. Lett. 87, 093001 (2001).

[21] C.A. Ullrich and W. Kohn, Phys. Rev. Lett. 89, 156401
(2001).

[22] T. Grabo, T. Kreibich, S. Kurth, and E.K.U. Gross, in Strong Coulomb Correlations in Electronic Structure Calculations: Beyond Local Density Approximations, edited by V. Anisimov (Gordon and Breach, Amsterdam, 2000), p. 203.

[23] J. Krieger, Y. Li, and G. Iafrate, Phys. Rev. A 46, 5453 (1992).

[24] S. Ivanov, S. Hirata, and R.J. Bartlett, Phys. Rev. Lett. 83, 5455 (1999).

[25] A. Görling, Phys. Rev. Lett. 83, 5459 (1999).

[26] M. Städele, J.A. Majewski, P. Vogl, and A. Görling, Phys. Rev. Lett. 79, 2089 (1997).

[27] S. Kümmel and J.P. Perdew, Phys. Rev. Lett. 90, 043004 (2003).

[28] S. Kümmel and J.P. Perdew, Phys. Rev. B 68, 035103 (2003).

[29] J.B. Krieger, Y. Li, and G.J. Iafrate, Phys. Rev. A 45, 101 (1992).

[30] O.V. Gritsenko and E.J. Baerends, Phys. Rev. A 64, 42506 (2001).

[31] F. Della Sala and A. Görling, J. Chem. Phys. 115, 5718 (2001).

[32] M. Grüning, O.V. Gritsenko, and E.J. Baerends, J. Chem. Phys. 116, 6435 (2002).

[33] G. te Velde et al., J. Comput. Chem. 22, 931 (2001).

[34] E. Engel, in A Primer in Density Functional Theory, Vol. 620 of Lecture Notes in Physics, edited by C. Fiolhais, F. Nogueira, and M. Marques (Springer, Berlin, 2003), p. 1. 